\title{
Les problèmes de comportement à l'enfance et les problèmes dépressifs : rôle médiateur du rejet social, des interactions parent-enfant et des difficultés scolaires Childhood behaviour problems and depressed mood: The mediating role of social rejection, parent-child interaction and academic difficulty
}

\author{
Mélanie Lapalme, Katherine Picarou, Marie-Josée Letarte, Geneviève Paquette, \\ Michèle Déry et Pierrette Verlaan
}

Volume 47, numéro 1, 2018

URI : https://id.erudit.org/iderudit/1046773ar

DOI : https://doi.org/10.7202/1046773ar

Aller au sommaire du numéro

Éditeur(s)

Revue de Psychoéducation

ISSN

1713-1782 (imprimé)

2371-6053 (numérique)

Découvrir la revue

Citer cet article

Lapalme, M., Picarou, K., Letarte, M.-J., Paquette, G., Déry, M. \& Verlaan, P. (2018). Les problèmes de comportement à l'enfance et les problèmes dépressifs : rôle médiateur du rejet social, des interactions parent-enfant et des difficultés scolaires. Revue de psychoéducation, 47(1), 87-110.

https://doi.org/10.7202/1046773ar
Résumé de l'article

Les problèmes de comportement et les problèmes dépressifs surviennent fréquemment en cooccurrence à l'adolescence et entraînent leur lot de conséquences. En se basant sur le modèle de Patterson et Capaldi (1990), la présente étude a pour but de valider le rôle des variables de rejet social (rejet par les pairs, insertion sociale) et des difficultés scolaires, mais aussi des interactions parent-enfant (chaleur, coercition) en tant que médiateurs du lien entre les problèmes de comportement présents à l'enfance et le développement de problèmes dépressifs à l'aube de l'adolescence, et de vérifier si ces liens diffèrent selon le sexe. Au final, l'échantillon est composé de 675 enfants, dont 319 filles, âgés entre 6 et 9 ans initialement et recrutés parce qu’ils présentaient des problèmes de comportement ou différents facteurs de risque des problèmes de comportement. Les résultats montrent que, chez les garçons, c'est particulièrement la qualité de leur insertion sociale qui permet d'expliquer le lien entre la présence de problèmes de comportement et le développement de problèmes dépressifs ultérieurs. Chez les filles, c'est plutôt la qualité des interactions chaleureuse du parent envers elle. Sur le plan des retombées pratiques, les résultats pointent le rôle des relations parentales et de l'insertion sociale dont l'importance varie selon le sexe dans la relation entre les problèmes de comportement et la dépression. Certaines recommandations concernant les pratiques probantes en matière d'intervention auprès des enfants présentant des problèmes de comportement sont formulées.
Tous droits réservés (C) La Corporation de la Revue Canadienne de Psycho-Éducation, 2018
Ce document est protégé par la loi sur le droit d'auteur. L’utilisation des services d’Érudit (y compris la reproduction) est assujettie à sa politique d'utilisation que vous pouvez consulter en ligne.

https://apropos.erudit.org/fr/usagers/politique-dutilisation/ 


\title{
Les problèmes de comportement à l'enfance et les problèmes dépressifs : rôle médiateur du rejet social, des interactions parent-enfant et des difficultés scolaires
}

\author{
Childhood behaviour problems and depressed mood: \\ The mediating role of social rejection, parent-child interaction \\ and academic difficulty
}

\section{Lapalme ${ }^{1}$ \\ K. Picarou ${ }^{1}$ \\ M-J Letarte ${ }^{1}$ \\ G. Paquette ${ }^{1}$ \\ M. Déry ${ }^{1}$ \\ P. Verlaan ${ }^{1}$}

1 Groupe de recherche sur les inadaptations sociales de l'enfance, Département de Psychoéducation, Université de Sherbrooke

\section{Correspondance :}

Mélanie Lapalme

Groupe de recherche sur les inadaptations sociales de l'enfance

Département de Psychoéducation Université de Sherbrooke 2500 boul. de l'Université Sherbrooke (Québec) Canada J1K 2R1

Melanie.Lapalme@

USherbrooke.ca

Tél. : (819) 821-8000, ext. 62565

Fax: (819) 821-7235
Résumé

Les problèmes de comportement et les problèmes dépressifs surviennent fréquemment en cooccurrence à l'adolescence et entraînent leur lot de conséquences. En se basant sur le modèle de Patterson et Capaldi (1990), la présente étude a pour but de valider le rôle des variables de rejet social (rejet par les pairs, insertion sociale) et des difficultés scolaires, mais aussi des interactions parentenfant (chaleur, coercition) en tant que médiateurs du lien entre les problèmes de comportement présents à l'enfance et le développement de problèmes dépressifs à l'aube de l'adolescence, et de vérifier si ces liens diffèrent selon le sexe. Au final, l'échantillon est composé de 675 enfants, dont 319 filles, âgés entre 6 et 9 ans initialement et recrutés parce qu'ils présentaient des problèmes de comportement ou différents facteurs de risque des problèmes de comportement. Les résultats montrent que, chez les garçons, c'est particulièrement la qualité de leur insertion sociale qui permet d'expliquer le lien entre la présence de problèmes de comportement et le développement de problèmes dépressifs ultérieurs. Chez les filles, c'est plutôt la qualité des interactions chaleureuse du parent envers elle. Sur le plan des retombées pratiques, les résultats pointent le rôle des relations parentales et de l'insertion sociale dont l'importance varie selon le sexe dans la relation entre les problèmes de comportement et la dépression. Certaines recommandations concernant les pratiques probantes en matière d'intervention auprès des enfants présentant des problèmes de comportement sont formulées.

Mots-clés: problèmes de comportement extériorisés, problèmes dépressifs, médiation, rejet des pairs, insertion sociale, interactions parent-enfant, difficultés scolaires, différences selon le sexe 


\begin{abstract}
Behaviour problems and depressed mood often occur concurrently in adolescence and have various consequences. Drawing on the Patterson and Capaldi (1990) model, the present study sought to validate the role of two of its variables-social rejection (peer rejection, social insertion) and academic difficulty-and of another variable-parent-child interaction (warmth, coercion) - as mediators of the relationship between childhood behaviour problems and development of depressed mood in early adolescence. The study also sought to verify whether the role of these variables differed by gender. The final sample was composed of 675 children, including 319 girls, 6 to 9 years of age at study outset. They were recruited for presenting behaviour problems or different risk factors for behaviour problem. The results showed that, for boys, quality of social insertion in particular allowed explaining the relationship between presence of behaviour problems and later development of depressed mood. For girls, instead, it was quality of warm parent-child interaction. In terms of practical application, the results underscore the gender-specific mediating role of parent-child interaction and of social insertion in the relationship between childhood behaviour problems and depressed mood. Recommendations are made regarding evidence-based practices for intervening with children with behaviour problems.
\end{abstract}

Keywords: externalizing behaviour problems, depressive problems, mediation, peer rejection, social insertion, parent-child interaction, academic difficulty

\title{
Introduction
}

Les enfants qui présentent des problèmes de comportement courent un risque accru de développer aussi des problèmes dépressifs (American Psychological Association, APA, 2013; Zahn-Waxler, Shirtcliff, Marceau, 2008). Si les problèmes de comportement et les problèmes dépressifs entraînent chacun leur lot de conséquences, leur cooccurrence apporte des impacts négatifs encore plus importants sur le fonctionnement personnel et social des enfants à court et à moyen terme (Reinecke, 1995; Wolff et Ollendick, 2006). Une meilleure compréhension des médiateurs impliqués dans le développement des problèmes dépressifs selon le sexe, plus particulièrement lorsqu'ils succèdent aux problèmes de comportement, permettra éventuellement d'intervenir de manière plus ciblée pour prévenir la survenue de problèmes dépressifs.

Les problèmes de comportement, notamment extériorisés, sont dirigés vers l'environnement physique ou vers autrui (Kazdin, 2005) et sont représentés par de l'agressivité, un manque d'obéissance, de l'irritabilité, de l'argumentation ou des comportements antisociaux (Roskam, Kinoo et Nassogne, 2007). Ils se manifestent donc principalement par les différents symptômes liés au trouble oppositionnel avec provocation ou au trouble des conduites (Arcelus et Vostanis, 2005; APA, 2013; King et al., 2005; Wolff et Ollendick, 2006;), lesquels comptent parmi les problèmes les plus fréquemment rencontrés chez les enfants et les adolescents (Arcelus et Vostanis, 2005; King et al., 2005; Wolff et Ollendick, 2006), affectant de $33 \%$ à $75 \%$ des enfants cliniquement référés (Déry, Toupin, Pauzé et Verlaan, 2004; Reinecke, 1995). En population générale, les prévalences varient de $1 \%$ à $11 \%$ selon l'âge et le sexe des enfants et selon le trouble spécifique présenté (APA, 2013). Ces problèmes entraînent d'importantes conséquences, comme des difficultés à s'entendre avec les adultes, un risque élevé d'être rejeté par les pairs et 
d'avoir des difficultés scolaires (Beekhoven et Dekkers, 2005; Déry, Toupin, Pauzé et Verlaan, 2005; Reinecke, 1995; Wolff et Ollendick, 2006) puis de développer d'autres troubles, tels que la dépression à l'adolescence (APA, 2013; Déry), qui deviennent souvent chroniques à l'âge adulte (Waschbusch, 2002). Plus les problèmes de comportement surviennent tôt, plus le pronostic est sombre et plus les difficultés persistent à l'adolescence et à l'âge adulte (Burke, Waldman, Lahey, 2010; Rowe, Costello, Angold, Copeland et Maughan, 2010).

Chez les enfants et les adolescents, les problèmes dépressifs se manifestent surtout par de l'irritabilité et de la tristesse (APA, 2013). La prévalence de la dépression à l'enfance est difficile à établir (APA, 2013), mais pourrait se situer autour de $3 \%$ (Brotman et al., 2006, dans APA, 2013). La prévalence de la dépression augmente considérablement à l'adolescence (Ingoldsby, Kohl, McMahon, Lengua, 2006; Keenan et Hipwell, 2005; Kessler, Avenevoli et Merikangas, 2001) variant de $14 \%$ à $25 \%$ selon la sévérité de la dépression prise en compte (Kessler et al., 2001). Cette prévalence, particulièrement à l'adolescence, est deux à trois fois plus élevée chez les filles que chez les garçons (Ingoldsby et al., 2006; Zahn-Waxler et al. 2006). Chez les enfants présentant des problèmes de comportement, le risque de développer des problèmes dépressifs est aussi plus grand pour les filles (Cook, Pflieger, Connell et Connell, 2015; Stringaris, Lewis et Maughan, 2014; ZahnWaxler et al., 2008) et celles-ci risquent de présenter une dépression plus sévère et plus persistante que les garçons (Keenan et Hipwell, 2005). Les enfants ayant à la fois des problèmes de comportement et des problèmes dépressifs répondent moins bien aux interventions et sont particulièrement à risque de développer des difficultés d'adaptation importantes ( $p$. ex. idéation suicidaire, comportements criminels à l'âge adulte; voir la recension de Wolff et Ollendick, 2006).

Le modèle de Patterson et Capaldi (1990) explique le développement des problèmes dépressifs chez des enfants présentant des problèmes de comportement. L'apport de ce modèle se situe dans l'identification de mécanismes individuels et sociaux impliqués dans le développement de la dépression, tout en considérant l'apport de vulnérabilités familiales impliquées initialement dans le développement des problèmes de comportement. Ainsi, en accord avec les autres modèles explicatifs du développement des problèmes de comportement (Lahey et Waldman, 2003; Lahey, Waldman, McBurnett, 1999; Moffitt, 1993, 2006), Patterson et Capaldi (1990; Patterson, 1986) soutiennent que l'interaction entre l'environnement familial (faible statut socio-économique, pratiques éducatives lacunaires et coercitives, nombreux stress) et les caractéristiques de l'enfant (tempérament difficile, impulsivité, faible tolérance à la frustration) prédisposent au rejet parental qui, à son tour, exacerbe les facteurs de risque déjà présents. Cette chaîne interactive contribuerait à l'apparition des problèmes de comportement extériorisés chez les enfants, parmi lesquels certains développeront des problèmes dépressifs. Le rejet parental, vu comme résultant d'interactions difficiles entre l'enfant et son environnement (Capaldi et Patterson, 1990; Patterson, 1986), pourrait jouer un rôle médiateur entre les problèmes de comportement et les problèmes dépressifs.

La relation entre les problèmes de comportement et les problèmes dépressifs a été démontrée à maintes reprises (Arcelus et Vostanis, 2005; Reinecke, 1995; Wolff et Ollendick, 2006; Zahn-Waxler et al., 2008). Par exemple, tel que 
rapporté par Wolff et Ollendick (2006), près de $50 \%$ des enfants présentant des problèmes de comportement développeraient aussi des problèmes dépressifs à l'adolescence et inversement, le tiers des adolescents dépressifs présenteraient aussi un trouble des conduites et la moitié, un trouble oppositionnel. Bien que les filles soient moins nombreuses que les garçons à présenter des problèmes de comportement, rappelons que lorsqu'elles en présentent, elles sont plus à risque de développer des problèmes dépressifs (Cook et al., 2015; Stringaris et al., 2014; Zahn-Waxler et al., 2008).

Patterson et Capaldi (1990) proposent trois catégories de médiateurs pour expliquer le lien entre les problèmes de comportement chez les enfants et les problèmes dépressifs ultérieurs : le rejet social, les difficultés scolaires et la faible estime de soi. Selon eux, les enfants présentant des problèmes de comportement courent un risque plus élevé de développer des difficultés scolaires, d'être rejetés par les pairs et les adultes et de recevoir peu de soutien de la part de leur entourage. Dans ce sens, nous croyons que la qualité des interactions parent-enfant, en plus des interactions avec les pairs, pourrait aussi jouer un rôle médiateur entre les problèmes de comportement et les problèmes dépressifs. Finalement, l'image que l'enfant a de lui-même serait influencée négativement par ses problèmes de comportement, l'amenant à se dévaloriser et diminuant son estime de soi, reconnue comme étant un précurseur de la dépression (Patterson et Capaldi, 1990), voire une manifestation de ce trouble (APA, 2013; Beck, Steer et Brown, 1996). Bien que ce modèle propose une explication plausible du lien entre les problèmes de comportement et les problèmes dépressifs, il a toutefois été construit à partir d'études empiriques réalisées uniquement auprès des garçons. Depuis, ce modèle est soutenu empiriquement par différentes études longitudinales (Burt et Roisman, 2010, van Lier, Vitaro, Barker, Brendgen, Tremplay et Boivin, 2012) traitant du rôle médiateur de divers indicateurs du rejet social et des difficultés scolaires sur les liens entre les problèmes de comportement à l'enfance et les problèmes intériorisés futurs. Ces études montrent des liens significatifs de médiation entre, d'une part, les problèmes extériorisés initiaux et, d'autre part, les difficultés scolaires (Burt et Roisman, 2010; van Lier et al., 2012) et les faibles compétences sociales (Burt et Roisman, 2010) deux ou trois ans plus tard. La victimisation comme médiateur entre les problèmes extériorisés et les problèmes intériorisés tend à être significative (van Lier et al., 2012). Dans l'ensemble, ces liens s'observeraient tant chez les garçons que chez les filles (Burt et Roisman, 2010; van Lier et al., 2012). Ces études sont intéressantes, mais ne concernent pas spécifiquement les problèmes dépressifs, mais plutôt l'ensemble des problèmes intériorisés incluant l'anxiété (Burt et Roisman, 2010; van Lier et al., 2012) et la somatisation (Burt et Roisman, 2010).

D'autres études traitant des facteurs associés directement aux problèmes dépressifs chez les enfants présentant des problèmes de comportement sont intéressantes. Les résultats de ces études appuient l'hypothèse selon laquelle différents indicateurs du rejet social, des difficultés scolaires, mais aussi du rejet parental, sont associés à ces deux problèmes. Les enfants, garçons et filles âgés de 11 à 13 ans, présentant simultanément des problèmes de comportement et des problèmes dépressifs, perçoivent recevoir moins de soutien social de la part de leurs parents, de leurs enseignants et de leurs pairs que les enfants présentant seulement l'un ou l'autre des problèmes (Rockhill, Stoep, McCauley, Katon, 2009) 
et sont moins bien intégrés socialement (Ezpeleta Granero, Doménech, 2005; Fanti et Henrich, 2010). Dans le même sens, les enfants (11-14 ans) présentant à la fois des problèmes dépressifs sévères et des problèmes de comportements sévères seraient moins compétents socialement et, sur le plan du rejet parental, ces enfants vivraient plus de conflits avec leurs parents; ces derniers seraient également moins impliqués auprès d'eux que les enfants présentant un niveau moins sévère de l'un ou l'autre des problèmes (Chen et Simons-Morton, 2009). Ces résultats s'appliqueraient tant aux garçons qu'aux filles, les garçons ayant toutefois davantage de conflits avec leurs parents. Enfin, Evelien, Van Lier, Stegge, Terwogt et Koot (2011) étudient le rôle médiateur du rejet par les pairs dans la relation entre les problèmes de comportement mesurés à l'âge de 5 ans et les problèmes dépressifs dans les deux années suivantes. Leurs résultats indiquent que les problèmes de comportement entraînent le rejet par les pairs, qui à son tour, augmente le risque de développer des problèmes dépressifs et cela, autant chez les garçons que chez les filles.

Sur le plan des difficultés scolaires, les résultats des études soutiennent aussi le modèle de Patterson et Capadi (1990). Ainsi, les enfants présentant une trajectoire marquée à la fois par des problèmes dépressifs et comportementaux importants obtiennent des résultats scolaires plus faibles et cumulent plus d'échecs scolaires que ceux qui ont des problèmes dépressifs ou comportementaux moins sévères (Chen et Simons-Morton, 2009). Par ailleurs, les problèmes d'apprentissage et la reprise d'une année scolaire à l'adolescence augmentent le risque de présenter simultanément des problèmes dépressifs et des problèmes de comportements (Ezpeleta et al., 2005). Ces résultats sur les difficultés scolaires s'appliquent tant aux garçons qu'aux filles (Ezpeleta et al., 2005), bien que les garçons vivent plus d'échecs scolaires que les filles (Chen et Simons-Morton, 2009).

Dans l'ensemble, les études mettent en évidence plusieurs indicateurs pertinents susceptible d'appuyer le modèle de Patterson et Capaldi (1990). Par exemple, sur le plan du rejet social, il semble que le rejet par les pairs soit important (Evelien et al., 2001), mais aussi la qualité de son insertion sociale (Ezpeleta et al., 2005; Fanti et Henrich, 2010). Dans une conceptualisation élargie du modèle, la qualité des interactions parent-enfant, que cela concerne l'implication, le soutien ou la présence de conflits (Chen et Simons-Morton, 2009; Rockhill et al., 2009), serait aussi à considérer. II semble donc important d'étudier simultanément plusieurs indicateurs de rejet social et parental, en plus des difficultés scolaires, afin de clarifier le rôle de chacun. De plus, mise à part l'effet médiateur du rejet par les pairs étudié par Evelien et al. (2011), l'effet de médiation des autres indicateurs n'a pas été vérifié dans ces études. De plus, elles montrent qu'ils sont associés à la cooccurrence simultanée des problèmes dépressifs et extériorisés, et non à la cooccurrence successive de ces deux problèmes.

La majorité des études ont été réalisées sur des échantillons issus de la population générale (Chen et Simons-Morton, 2009; Fanti et Henrich, 2010; Evelien et al., 2011; Rockhill et al., 2009). Or, la prévalence des troubles de comportement et de la dépression est beaucoup plus faible dans la population générale que dans la population clinique et les problèmes présentés sont moins sévères. On peut donc s'interroger sur le nombre d'enfants, et particulièrement le nombre de 
filles, présentant réellement des problèmes de comportement et des problèmes dépressifs dans ces études. Les résultats de ces études quant aux facteurs associés à la présence de problèmes de comportement et de problèmes dépressifs chez des enfants qui n'en présentent peut-être pas, devraient donc être interprétés avec nuances.

S'inspirant du modèle de médiation de Patterson et Capaldi (1990), la présente étude vérifie, à partir de différents indicateurs, si le rejet social, parental et les difficultés scolaires expliquent comment les enfants qui présentent des problèmes de comportement, un sous-groupe particulièrement à risque (APA 2013; Zahn-Waxler et al., 2008), en viennent ultérieurement à présenter des problèmes dépressifs. Plus précisément, elle vérifie si la relation entre la sévérité des problèmes de comportement déjà présents entre l'âge de 6 à 8 ans, et les problèmes dépressifs quatre ans plus tard s'explique par l'effet médiateur du rejet par les pairs, de la qualité de son insertion sociale, des interactions chaleureuses ou coercitives avec son parent ainsi que des difficultés scolaires. Cette étude vérifie également si les mécanismes explicatifs sont les mêmes pour les garçons et pour les filles (invariance du sexe). Considérant les résultats des études recensées et surtout en s'appuyant sur le modèle de Patterson et Capaldi (1990), on peut poser l'hypothèse que, la présente étude confirmera l'association entre la sévérité des problèmes de comportement à l'enfance et la sévérité des problèmes dépressifs à l'aube de l'adolescence. Mais encore, on s'attend à ce que plus les enfants présenteront des problèmes de comportement sévères à l'enfance, plus ils seront rejetés par leurs pairs deux ans plus tard et auront des difficultés scolaires, et plus leurs problèmes dépressifs à l'adolescence seront sévères. Les questions touchant la qualité de l'insertion sociale, les indicateurs du rejet parental et l'invariance du sexe sont plutôt exploratoires.

En plus d'étudier un modèle de médiation incluant plusieurs médiateurs potentiels, cette étude a recours à un échantillon constitué de nombreux enfants (particulièrement de filles) qui présentent des problèmes de comportement. Une meilleure connaissance des facteurs impliqués dans la cooccurrence successive entre les problèmes de comportement et les problèmes dépressifs permettra, éventuellement, de prévenir l'exacerbation des difficultés présentes à l'enfance et ainsi éviter le développement de problèmes d'adaptation plus complexes à l'adolescence, à risque de chronicité à l'âge adulte.

\section{Méthode}

\section{Participants}

L'échantillon de départ est composé de 744 enfants ( $46,7 \%$ de filles) âgés de 6 à 9 ans $(M=8,4$ ans; é.t. $=0,9)$. La plupart de ces enfants $(n=339$ enfants, $41 \%$ filles) ont été recrutés à partir des listes d'élèves qui reçoivent des services de l'école pour des problèmes de comportement. Ces élèves sont issus de huit commissions scolaires de quatre régions du Québec (Québec, Estrie, Montérégie, Montréal). Suivant la procédure établie au ministère de l'Éducation, les enfants qui ont des difficultés de comportement sont signalés par leur enseignant aux services scolaires complémentaires, et ce n'est qu'après une période d'observation et 
une évaluation formelle par un professionnel que la présence de problèmes de comportement est confirmée. À partir de ces listes d'élèves, toutes les filles de moins de 10 ans, et environ un garçon sur quatre, choisi au hasard, ont été ciblés pour participer à l'étude (taux de participation : $75,1 \%$ ) en trois vagues $(2008,2009$, 2010). Le taux de participation est similaire selon le sexe, le niveau scolaire et l'indice de défavorisation des écoles (indice en 10 points calculé par le Ministère). Pour uniformiser l'évaluation des problèmes de comportement, tous les enfants ont été réévalués dans le cadre de l'étude avec les échelles orientées sur les critères du Diagnostic and statistical manual of mental disorders (DSM) pour le trouble des conduites (TC) et le trouble de l'opposition avec provocation (TOP) (Achenbach et Rescorla, 2001). Les échelles ont été complétées par un parent et par l'enseignant. Pour être retenu dans l'échantillon, le score de difficulté des enfants devait être au-dessus du seuil de risque élevé à l'une ou l'autre des échelles (score $T>65$ ). Le score moyen des enfants retenus est de 75,3 (é.t. $=6,7$ ), ce qui est au-dessus du seuil clinique de 70 (> 98e percentile). II n'y a pas de différences selon le sexe.

Comme il est reconnu que les filles présentant des difficultés comportementales peuvent être moins souvent signalées que les garçons, en particulier en milieu scolaire (Bradshaw, Schaeffer, Petras et lalongo, 2010; Coutinho, Oswald, Best et Forness, 2002; Costello, Sampson, Kessler et Merikangas, 2014), une stratégie de recrutement complémentaire a été utilisée en 2009 pour identifier les enfants susceptibles de présenter des problèmes de comportement, mais ne recevant pas de services du milieu scolaire. Cette stratégie complémentaire de recrutement des enfants avec des problèmes de comportement a consisté en un repérage systématique par étape (Lane, 2003). D'abord, les difficultés de comportement des élèves ont été évaluées à partir d'échelles simplifiées et plus sensibles par l'enseignant et les parents. Si des difficultés étaient détectées, une évaluation plus approfondie était faite à l'aide des mêmes échelles orientées sur les critères du DSM afin d'uniformiser l'évaluation des problèmes de comportement. Cette stratégie a été appliquée dans 99 classes, de différentes écoles ayant un indice de défavorisation relativement élevé (> 7) afin d'augmenter la probabilité de détection d'élèves avec des problèmes de comportement et de ne pas creuser trop d'écarts avec les élèves recrutés avec la première stratégie. Cette stratégie a permis d'identifier 95 autres enfants répondant aux critères de sélection, dont $58 \%$ de filles, ce qui conforte sa valeur complémentaire. Le score moyen de difficultés comportementales de ces enfants $(M=70,8$; é.t. $=4,9)$ est légèrement inférieur à celui des enfants signalés, mais demeure au-dessus du seuil clinique. II n'y a pas de différence selon le sexe.

Enfin, 310 enfants $(49,7 \%$ de filles) ne présentant pas de problèmes de comportement ont été recrutés dans les mêmes écoles. Environ $25 \%$ de ces enfants ont été sélectionnés de manière aléatoire parmi ceux ayant participé à la procédure de repérage systématique et n'ayant pas de difficultés particulières. Les autres enfants ont été recrutés au hasard parmi les listes d'élèves réguliers âgés de moins de 10 ans dans les écoles à indice de défavorisation élevé. L'ensemble de ces enfants ne se différencie pas de ceux avec des problèmes de comportement sur l'âge moyen. Ils ont tous un score de difficultés inférieur à 65. Le score T moyen de problèmes de comportement est de 56,9 (é.t. $=4,4$ ), ce qui se situe dans la zone de normalité. Ces différentes stratégies de recrutement ont été utilisées afin 


\section{4}

de mieux représenter l'ensemble des enfants d'âge scolaire primaire présentant ou non des difficultés comportementales. Comme les problèmes dépressifs sont prédits 4 ans plus tard, et que le taux d'attrition entre le premier et le troisième temps de mesure est de $10 \%$, au final, les analyses ont été réalisées auprès de 675 enfants, dont 319 filles. Parmi ces 675 enfants, 87 (13\%) obtiennent un score de problèmes dépressifs se situant au-dessus du seuil de risque élevé (score $T \geq$ 65) au troisième temps de mesure.

\section{Variables mesurées et instruments de mesure}

L'étude opte pour un devis corrélationnel prédictif utilisant les données issues de trois temps de mesure sur 4 ans. D'abord, la sévérité des problèmes de comportement (prédicteur) au premier temps de mesure (T1) a été utilisée. Ensuite, les variables médiatrices, soit le rejet parental (chaleur, coercition), le rejet social (rejets par les pairs, qualité de l'insertion sociale) et les difficultés scolaires de l'enfant telles que mesurées deux ans plus tard (deuxième temps de mesure, T2) ont été utilisés. Quant à la variable prédite, soit la sévérité des problèmes dépressifs, elle a été mesurée quatre ans plus tard (troisième temps de mesure, T3). De plus, l'âge au T1 a été contrôlé, ainsi que le statut initial (T1) des variables médiatrices et de la variable prédite (voir la figure 1).

Problèmes dépressifs. La sévérité des problèmes dépressifs a été mesurée par l'Achenbach System of Empirically Based Assessment (ASEBA; Achenbach et Rescorla, 2001), un outil permettant d'évaluer les problèmes de comportement de nature intériorisée et extériorisée auprès des enfants âgés de 6 à 18 ans selon différents répondants (parent, adolescent, enseignant). Le portrait comportemental de l'enfant est établi à partir d'une série d'items répondus sur une échelle de type Likert avec trois choix de réponse : " pas du tout vrai », " quelques fois vrai » ou « vrai ou souvent vrai ». L'outil permet l'utilisation d'échelles d'orientation DSM qui regroupent des items correspondant à certaines manifestations de troubles mentaux. Pour cette étude, le score standardisé à l'échelle d'orientation DSM des troubles affectifs se rapportant à la présence de symptômes dépressifs (p. ex., manque d'énergie, est déprimé, trouve qu'il est sans valeur) rapportés par le parent sera utilisé afin de contrôler pour la dépression au T1. Un score moyen standardisé à l'échelle d'orientation DSM des troubles affectifs de la version parent et de la version autorapportée par l'adolescent sera utilisé pour prédire les problèmes dépressifs au T3. Plus le score moyen est élevé, plus la sévérité de la dépression augmente. Les scores obtenus aux échelles sont standardisés selon l'âge, le sexe et le répondant.

En termes de qualités psychométriques, l'échelle d'orientation DSM pour les troubles affectifs présente une corrélation de fidélité test-retest de 0,80 pour la version autorapportée et de 0,84 pour la version rapportée par le parent (Achenbach et Rescorla, 2001). Cette échelle ne tient pas compte de tous les symptômes de la dépression, mais d'un point de vue dimensionnel, elle s'avère être un indice appréciable de la sévérité de la dépression auprès des enfants et des adolescents (Achenbach et Rescorla, 2001). Au sein de l'échantillon de la présente étude, le coefficient de l'alpha de Cronbach est de 0,81. 
Problèmes de comportement. La sévérité des problèmes de comportement a aussi été mesurée à partir de l'ASEBA (Achenbach et Rescorla, 2001). Le score à l'échelle de problèmes extériorisés rapporté par le parent qui regroupe deux sous-échelles, soit celles des comportements de bris de règle ( $p$. ex. mentir, vandalisme, vol) et des comportements agressifs ( $p$. ex. être désobéissant, détruire le bien d'autrui, argumenter), a été utilisé. Plus le score à l'échelle des problèmes extériorisés est élevé, plus les problèmes de comportement sont sévères. Le score obtenu est standardisé selon l'âge, le sexe et le répondant. En termes de qualités psychométriques, l'échelle des problèmes extériorisés rapportés par le parent présente une corrélation de fidélité test-retest de 0,92 pour un intervalle de huit jours (Achenbach et Rescorla, 2001). Dans la présente étude, le coefficient de l'alpha de Cronbach obtenu pour la version parent est de 0,93.

Rejet parental. Les interactions chaleureuses et coercitives entre le parent et l'enfant ont été évaluées à l'aide du Perceived Parental Acceptance-Rejection Questionnaire (PARQ; Rohner, 2005) complété par le parent. Ce questionnaire est composé de 60 items répondus à partir d'échelle de type Likert à quatre choix de réponse : "presque toujours vrai (1) ", " parfois vrai (2) ", " Rarement vrai (3) » ou «presque jamais vrai (4) ». Deux échelles du questionnaire ont été utilisées pour mesurer la qualité des interactions parent-enfant : la chaleur et la coercition (hostilité/rejet). Plus les scores sont élevés, plus le parent est chaleureux envers son enfant ou plus il est coercitif. Une méta-analyse réalisée par Khaleque et Rohner (2001) regroupant les études ayant utilisé le PARQ a permis de calculer un coefficient de l'alpha de Cronbach de 0,89 , ce qui indique une excellente cohérence interne. De plus, toutes les études ayant effectué une analyse de la fidélité testretest furent regroupées, afin d'obtenir un coefficient moyen de 0,62, et ce, pour des test-retest effectués à des intervalles variant de trois semaines à sept ans (Khaleque et Rohner, 2001). Dans la présente étude, les coefficients de l'alpha de Cronbach sont de 0,83 pour la sous-échelle "Chaleur » et de 0,87 pour la souséchelle "Coercition », indiquant aussi une très bonne cohérence interne.

Rejet social. D'abord, le rejet par les pairs a été mesuré par trois items tirés du Teacher Peer Social Skills (Dishion et Kavanagh, 2003). Cet outil permet de mesurer le nombre de pairs qui rejettent, aiment ou ignorent l'enfant cible. Les items sont répondus sur échelle de type Likert à cinq choix de réponse : " très peu (1) », « peu (2) », « environ la moitié (3) », « la plupart (4) » ou « presque tous (5) ». Plus le score est élevé, plus l'enfant est rejeté par une grande proportion de ses camarades de classe. La cohérence interne des trois items utilisés présente un coefficient de l'alpha de Cronbach de 0,82 dans la présente étude.

Quant à la qualité de l'insertion sociale de l'enfant, elle est mesurée par différents items tirés du Child Behavior Checklist (CBCL; Achenbach et Edelbrock, 1983) adaptée pour l'Enquête québécoise sur la santé mentale des jeunes (Valla, Bergeron, Breton, Gaudet, Berthiaume et al., 1997). Ces items permettent de documenter le nombre d'amis proches de l'enfant, le nombre moyen de fois où il fait des activités avec eux durant la semaine, ainsi que le nombre d'organisations sociales ou sportives dont il fait partie. À partir de ces différents items, un score reflétant la qualité de l'insertion sociale de l'enfant est calculé. Plus le score est élevé, plus l'enfant est intégré socialement. 
Difficultés scolaires. Les difficultés scolaires de l'enfant sont mesurées par différents items tirés de l'Academic Performance Rating Scale (APRS ; DuPaul, Rapport et Perriello, 1991) complété par l'enseignant. Ces items portent sur les résultats scolaires de l'enfant et sur ses habiletés d'apprentissage et permettent le calcul d'un score total de performance scolaire. L'enseignant doit répondre à partir de différents choix de réponse (par exemple : avec quelle facilité cet enfant maîtrise-t-il la nouvelle matière; comment peut-on qualifier les habiletés en lecture de cet enfant; l'enfant commence-t-il (elle) son travail avant d'avoir compris les consignes, etc.). Un faible score total indique que l'enfant présente de nombreuses difficultés. L'outil présente de bonnes qualités psychométriques avec un coefficient de corrélation pour la fidélité test-retest à une semaine d'intervalle de 0,95 pour le score total de difficultés scolaires. Cette échelle a aussi démontré une bonne validité discriminante entre les enfants qui présentent des difficultés et ceux qui n'en présentent pas (Dupaul et al., 1991). Pour la présente étude, l'outil présente une excellente cohérence interne avec un coefficient de l'alpha de Cronbach de 0,88.

\section{Déroulement}

Les parents des élèves ciblés ont été contactés par les intervenants des écoles ou par les assistants de recherche afin d'être informés sur le projet. À la suite de la présentation du projet, les parents ont signé le formulaire de consentement approuvé par le comité d'éthique en Éducation et Sciences sociales de l'Université de Sherbrooke. Ce consentement inclut l'autorisation de colliger des informations auprès de l'enseignant de l'enfant. Le consentement verbal de l'enfant a aussi été obtenu. L'enfant cible et le parent ont ensuite été rencontrés séparément à domicile par un assistant de recherche pour une entrevue d'environ 90 minutes afin de remplir les questionnaires. Les enseignants ont rempli les questionnaires (environ 30 minutes) soit par envoi postal, soit en entrevue téléphonique avec un assistant de recherche. Seuls 1,3\% des enseignants ont refusé de participer à l'étude et de répondre aux différents questionnaires. Le parent, l'enfant et l'enseignant ont reçu une compensation financière ou matérielle pour leur participation à chacun des temps de mesure.

\section{Analyses}

Une analyse de médiation a été réalisée à partir du logiciel Mplus 7.2 (Muthén et Muthén, 1998-2014) afin de tester la contribution des variables médiatrices sur le lien entre les problèmes extériorisés au T1 et les problèmes dépressifs au T3 (voir la figure 1 pour le modèle de médiation de base). Le lien $a$ détermine l'association (lien direct) entre les problèmes extériorisés et le médiateur, tandis que le lien $b$ illustre le lien direct entre le médiateur et les problèmes dépressifs. Le lien $c$ ' réfère au lien direct entre la variable indépendante (sévérité des problèmes extériorisés) et la variable dépendante (sévérité des problèmes dépressifs) en contrôlant pour les médiateurs. Enfin, les liens indirects (spécifiques et total), qui correspondent au produit entre $a$ et $b$, illustre la présence de médiation ou non. Le seuil de signification utilisé est le seuil habituel de $p<0,05$. 
Un seul modèle, incluant les cinq médiateurs, a été analysé. L'effet modérateur du sexe a été testé par invariance de modèles. Un modèle où les paramètres sont nécessairement égaux selon le sexe (modèle contraint) a été testé suivi d'un deuxième modèle où les paramètres sont libres de varier selon le sexe (modèle variant) et leur $\mathrm{khi}^{2}$ a été comparé (test d'invariance). Dans le cas où la différence entre les $\mathrm{khi}^{2}$ s'avère significative, le modèle doit être considéré comme variant selon le sexe et les paramètres doivent être estimés séparément pour chaque sexe (méthode en sous-groupes). Les différents indices retenus pour juger de la qualité des modèles sont le Root mean square error of approximation (RMSEA), le Maximum likelihood ratio test statistic $\left(\mathrm{khi}^{2}\right)$ et le pourcentage de variance expliquée par le modèle selon le Comparative fit index (CFI). Hu et Bentler (1999) suggèrent d'utiliser comme critère de qualité un RMSEA $<0,06$ et un CFI idéal $>0,96$ et minimalement de 0,9. Pour la valeur du $\mathrm{khi}^{2}$, bien qu'il soit grandement influencé par la taille de l'échantillon, cette valeur doit idéalement être suffisamment petite pour ne pas atteindre le seuil de signification de $p<0,05$ (Bollen, 1989). Le logiciel utilisé (Mplus 7.2, Muthén et Muthén, 1998-2014) s'accommode des données manquantes et permet l'utilisation du bootstrapping qui est une technique robuste lorsque les variables ne se distribuent pas normalement.

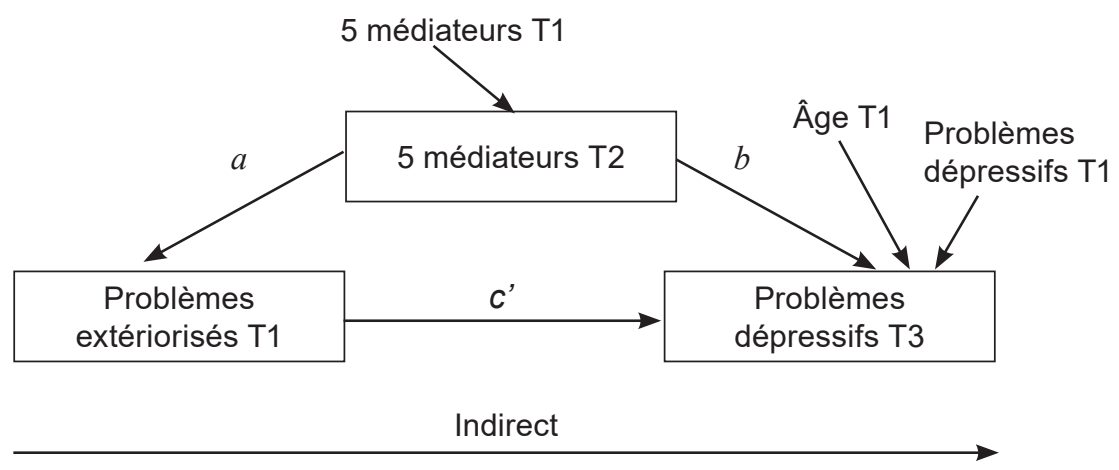

Figure 1. Illustration du modèle de médiation de base

\section{Résultats}

\section{Données corrélationnelles et descriptives}

Tel qu'attendu, les corrélations (voir le tableau 1) entre les problèmes de comportement extériorisés au T1 et les problèmes dépressifs au T3 s'avèrent significatives et positives pour les garçons $(r=0,42)$ et pour les filles séparément $(r=0,61)$. Les corrélations élevées et significatives entre le T1 et le T2 pour chaque médiateur testé (variant de $r=0,35$ et $r=0,65$ chez les garçons et de $r=0,45$ à $r=0,71$ chez les filles) montrent la nécessité de contrôler pour leur mesure initiale dans chaque modèle. De plus, les corrélations entre la mesure des problèmes dépressifs du T1 et celle du T3 (garçons : $r=0,49$; fille : $r=0,43$ ) indiquent aussi la nécessité de contrôler pour la sévérité initiale des problèmes dépressifs. Bien 
que l'âge ne soit significativement corrélé avec aucune variable à l'étude chez les garçons et avec une seule variable chez les filles, par prudence, l'âge des enfants au T1 a été contrôlé dans le modèle. Comme toutes les corrélations entre la variable indépendante et les médiateurs et entre les médiateurs et la variable dépendante sont significatives, il est possible de tester les effets de médiations. Le tableau 2 présente les données descriptives (moyenne, écart-type) pour l'ensemble de l'échantillon, ainsi que pour les garçons et les filles séparément. Les garçons présentent davantage de problèmes extériorisés au T1 et dépressifs au T3 que les filles et ont plus de difficultés scolaires (plus le score est faible, moins bonnes sont les performances et plus les difficultés sont importantes) aux T1 et T2. Malgré le peu de différences observées entre les garçons et les filles, la différence entre le khi ${ }^{2}$ du modèle contraint (sexes égaux) et du modèle libre (variant selon le sexe) s'avère significative (voir le tableau 3). Ainsi, dans le modèle incluant les cinq médiateurs, la méthode en sous-groupe a été utilisée et les coefficients standardisés ont été estimés pour les filles et les garçons séparément.

\section{Effets de médiation}

Les indices pour juger de la qualité du modèle initial (sans égard au sexe) et du modèle variant selon le sexe (méthode en sous-groupe) sont illustrés dans le tableau 3. Tant le modèle de médiation initial que le modèle variant selon le sexe répondent bien aux critères de qualité exigés (Hu et Bentler, 1999; Bollen, 1989). Chaque variable, prise individuellement, contribue à expliquer un pourcentage de variance (R2) considérable dans le modèle (voir le tableau 4). Les liens directs entre les problèmes extériorisés au T1 et les médiateurs, et entre les médiateurs et les problèmes dépressifs au T3, sont présentés, pour les filles et les garçons, sur les tracés de la figure 2. Les liens indirects (effet de médiation) pour chaque médiateur et pour le modèle total sont présentés dans le tableau 4. Pour l'ensemble du modèle, l'effet de médiation total s'avère significatif tant pour les garçons que pour les filles. Chez les garçons, comme le lien direct entre les problèmes extériorisés au T1 et les problèmes dépressifs au T3 n'est plus significatif une fois les médiateurs inclus dans le modèle, l'effet de médiation semble complet. Chez les filles, le lien entre les problèmes de comportement extériorisés au T1 et les problèmes dépressifs au T3 s'expliquerait, en partie (effet de médiation partiel), par l'ensemble des médiateurs du T2.

Plus spécifiquement, les résultats montrent plusieurs liens directs significatifs chez les garçons. Plus les problèmes extériorisés au T1 des garçons sont sévères, moins les interactions parent-enfant sont chaleureuses, plus elles sont coercitives, moins ils sont insérés socialement, plus ils sont rejetés par les pairs et moins ils performent à l'école. De plus, plus les interactions parent-enfant sont coercitives, et moins ils sont insérés socialement, plus leurs problèmes dépressifs au T3 sont sévères. Malgré ces nombreux liens directs significatifs, la qualité de l'insertion sociale est le seul médiateur significatif dans le modèle pour les garçons. Donc, chez les garçons, le lien entre les problèmes de comportement extériorisés au T1 et les problèmes dépressifs au T3 s'expliquerait surtout par la qualité de leur insertion sociale. 
Chez les filles, le lien de médiation s'expliquerait particulièrement par une interaction parent-enfant moins chaleureuse. Donc, plus les problèmes extériorisés sont sévères, moins le parent fait preuve de chaleur dans sa relation avec son enfant et plus les problèmes dépressifs au T3 sont sévères. II s'agit du seul lien indirect significatif chez les filles. Les résultats montrent aussi que plus les problèmes extériorisés au T1 sont sévères, plus les interactions parent-enfant sont coercitives et plus les filles sont rejetées par leurs pairs. Malgré ces liens directs significatifs, il ne s'agit pas médiateurs significatifs dans le modèle.

\section{Discussion}

En se basant sur le modèle de Patterson et Capaldi (1990), la présente étude avait pour but de valider le rôle des indicateurs de rejet social et des difficultés scolaires, mais aussi du rejet parental, comme médiateurs du lien entre les problèmes de comportement extériorisés présents à l'enfance et le développement de problèmes dépressifs à l'aube de l'adolescence. C'est en adoptant une conception élargie du modèle que nous avons inclus la qualité des interactions parent-enfant et plus particulièrement la chaleur et la coercition, comme indicateurs de rejet parental.

Le modèle étant initialement conceptualisé auprès des garçons (Patterson et Capaldi, 1990), la présente étude a l'avantage de vérifier l'application du modèle, du moins en ce qui concerne les indicateurs étudiés, auprès des garçons et des filles. Mais encore, l'étude a été réalisée auprès d'un large échantillon parmi lesquels un nombre important d'enfants, dont presque la moitié de filles, présentent un niveau cliniquement significatif de problèmes de comportement extériorisés. D'ailleurs, la combinaison de deux stratégies de recrutement a permis de sélectionner l'ensemble des enfants d'âge scolaire primaire présentant des difficultés comportementales à intensité variable et ce, qu'ils reçoivent des services complémentaires du milieu scolaire ou non. Ce large échantillon a permis de tester un même modèle de médiation incluant tous les indicateurs et d'utiliser la méthode en sous-groupe pour mieux déterminer les médiateurs les plus importants pour les garçons que pour les filles séparément.

D’abord, les résultats ont confirmé le lien déjà clairement établi entre les problèmes extériorisés et la présence de problèmes dépressifs (Reinecke, 1995; Arcelus et Vostanis, 2005; Zahn-Waxler et al., 2008; Wolff et Ollendick, 2006). En effet, les corrélations simples montrent que tant chez les garçons que chez les filles, plus la sévérité des problèmes de comportements extériorisés est importante au premier temps de mesure, plus les problèmes dépressifs sont élevés quatre ans plus tard. Globalement, les résultats montrent que l'ensemble des indicateurs (chaleur, coercition, insertion sociale, rejet par les pairs, difficultés scolaires) serait médiateur du lien entre les problèmes de comportement extériorisés et les problèmes dépressifs, mais que certains indicateurs seraient des médiateurs plus importants propres aux filles et propres aux garçons. 


\begin{tabular}{|c|c|c|c|c|c|c|c|}
\hline 7. & 8. & 9. & 10. & 11. & 12. & 13. & 14. \\
\hline 0,020 & $-0,013$ & 0,075 & 0,100 & $0,174^{* *}$ & 0,069 & $-0,044$ & 0,037 \\
\hline $0,415^{\star * *}$ & $0,405^{\star \star *}$ & $-0,296^{* * *}$ & $-0,209^{* * *}$ & $0,437^{* * *}$ & $0,378^{* * *}$ & $-0,478^{* * *}$ & $-0,398^{* * *}$ \\
\hline $0,322^{* * *}$ & $0,284^{* * *}$ & $-0,264^{* * *}$ & $-0,154^{* *}$ & $0,336^{\star * *}$ & $0,169^{* *}$ & $-0,369^{* * *}$ & $-0,255^{\star * *}$ \\
\hline $0,293^{* \star *}$ & $0,243^{* * *}$ & $-0,222^{* * *}$ & $-0,203^{* * *}$ & $0,329^{\star * *}$ & $0,301^{* * *}$ & $-0,363^{\star * *}$ & $-0,316^{* * *}$ \\
\hline$-0,580^{* * *}$ & $-0,382^{* * *}$ & 0,064 & 0,064 & $-0,214^{* * *}$ & $-0,185^{\star \star *}$ & $0,139^{*}$ & 0,096 \\
\hline$-0,448^{* * *}$ & $-0,500^{* * *}$ & $0,177^{* *}$ & $0,119^{*}$ & $-0,304^{* * *}$ & $-0,307^{* \star *}$ & $0,262^{* * *}$ & $0,254^{\star * *}$ \\
\hline- & $0,711^{* * *}$ & $-0,087$ & $-0,125^{\star}$ & $0,232^{\star * *}$ & $0,235^{\star * *}$ & $-0,177^{* *}$ & $-0,197^{* * *}$ \\
\hline $0,639^{* * *}$ & - & $-0,114^{*}$ & $-0,006$ & $0,240^{\star * *}$ & $0,244^{\star * *}$ & $-0,164^{\star *}$ & $-0,180^{* *}$ \\
\hline$-0,110^{*}$ & $-0,098$ & - & $0,449^{* * *}$ & $-0,207^{\star * *}$ & $-0,220^{\star \star *}$ & $0,289^{* * *}$ & $0,252^{* * *}$ \\
\hline$-0,148^{* *}$ & $-0,044$ & $0,362^{* * *}$ & - & $-0,176^{\star *}$ & $-0,174^{\star *}$ & $0,247^{* * *}$ & $0,208^{* * *}$ \\
\hline $0,148^{* *}$ & $0,152^{\star *}$ & $-0,258^{\star * *}$ & $-0,187^{\star * *}$ & - & $0,480^{* * *}$ & $-0,514^{* \star *}$ & $-0,351^{* * *}$ \\
\hline $0,151^{* *}$ & 0,075 & $-0,137^{*}$ & $-0,176^{* *}$ & $0,347^{* * *}$ & - & $-0,311^{* * *}$ & $-0,398^{* * *}$ \\
\hline$-0,217^{* * *}$ & $-0,136^{* *}$ & $0,198^{\star \star \star}$ & $0,174^{* * *}$ & $-0,410^{* * *}$ & $-0,251^{\star \star *}$ & - & $0,682^{\star \star \star}$ \\
\hline$-0,175^{\star \star}$ & $-0,064$ & $0,168^{* *}$ & $0,196^{* * *}$ & $-0,256^{\star * *}$ & $-0,366^{* * *}$ & $0,654^{* * *}$ & - \\
\hline
\end{tabular}

Note: Les corrélations pour les filles se trouvent au-dessus de la diagonale et les corrélations pour les garçons en dessous de la diagonale.

a Il s'agit d'une échelle inversée : plus le score est faible plus les difficultés scolaires sont importantes.

${ }^{*} p<, 05 ;{ }^{* *} p<, 01 ;{ }^{* * *} p<, 001$ 
Tableau 2. Présentation des données descriptives et comparaisons selon le sexe

\begin{tabular}{|c|c|c|c|c|c|}
\hline \multirow[t]{2}{*}{ Variables } & $\begin{array}{c}\text { Total } \\
(\mathrm{N}=675) \\
\end{array}$ & $\begin{array}{l}\text { Garçons } \\
(\mathrm{N}=356)\end{array}$ & $\begin{array}{c}\text { Filles } \\
(\mathrm{N}=319)\end{array}$ & \multirow[t]{2}{*}{ Étendu } & \multirow{2}{*}{$\begin{array}{c}\text { Valeur } \\
\text { de } T\end{array}$} \\
\hline & $M(e ́-t)$ & M (é.t.) & $M(e ́-t)$ & & \\
\hline Âge T1 & $8,40(0,93)$ & $8,40(0,95)$ & $8,36(0,92)$ & $6,3-10,6$ & 0,63 \\
\hline $\begin{array}{l}\text { Problèmes } \\
\text { extériorisés T1 }\end{array}$ & $62,71(10,62)$ & $63,72(9,91)$ & $61,91(11,19)$ & $33-86$ & $2,23^{*}$ \\
\hline $\begin{array}{l}\text { Problèmes } \\
\text { dépressifs T1 }\end{array}$ & $61,04(9,21)$ & $61,66(9,46)$ & $60,83(9,13)$ & $50-87$ & 1,16 \\
\hline $\begin{array}{l}\text { Problèmes } \\
\text { dépressifs T3 }\end{array}$ & $56,56(6,12)$ & $57,22(6,36)$ & $56,22(6,01)$ & $50-82$ & $2,02^{*}$ \\
\hline $\begin{array}{l}\text { Chaleur } \\
\text { parentale T1 }\end{array}$ & $68,87(4,14)$ & $69,08(4,11)$ & $68,64(3,99)$ & $41-72$ & 1,41 \\
\hline $\begin{array}{l}\text { Chaleur } \\
\text { parentale T2 }\end{array}$ & $69,35(3,73)$ & $69,49(3,63)$ & $69,29(3,68)$ & $45-72$ & 0,68 \\
\hline $\begin{array}{l}\text { Coercition } \\
\text { parentale T1 }\end{array}$ & $38,12(7,96)$ & $38,20(7,43)$ & $38,37(8,52)$ & $25-76$ & $-0,28$ \\
\hline $\begin{array}{l}\text { Coercition } \\
\text { parentale T2 }\end{array}$ & $37,16(7,99)$ & $37,76(7,99)$ & $36,59(8,08)$ & $25-66$ & 1,87 \\
\hline $\begin{array}{l}\text { Insertion } \\
\text { sociale T1 }\end{array}$ & $6,41(3,42)$ & $6,31(3,54)$ & $6,48(3,36)$ & $0-17$ & $-0,61$ \\
\hline $\begin{array}{l}\text { Insertion } \\
\text { sociale T2 }\end{array}$ & $6,33(3,47)$ & $6,27(3,53)$ & $6,24(3,43)$ & $0-18$ & 0,13 \\
\hline $\begin{array}{l}\text { Rejet } \\
\text { par les pairs T1 }\end{array}$ & $1,91(1,08)$ & $1,94(1,05)$ & $1,87(1,10)$ & $1-5$ & 0,82 \\
\hline $\begin{array}{l}\text { Rejet } \\
\text { par les pairs T2 }\end{array}$ & $2,03(1,13)$ & $2,09(1,09)$ & $1,98(1,16)$ & $1-5$ & 1,23 \\
\hline $\begin{array}{l}\text { Difficultés } \\
\text { scolaires T1a }\end{array}$ & $3,21(0,89)$ & $3,13(0,87)$ & $3,29(0,91)$ & $1,1-5$ & $-2,42^{*}$ \\
\hline $\begin{array}{l}\text { Difficultés } \\
\text { scolaires T2 }\end{array}$ & $3,12(0,82)$ & $2,98(0,78)$ & $3,26(0,84)$ & $1,2-5$ & $-4,23^{* * *}$ \\
\hline
\end{tabular}

a ll s'agit d'une échelle inversée : plus le score est faible plus les difficultés scolaires sont importantes.

${ }^{*} p<, 05 ;{ }^{* *} p<, 01 ;{ }^{* * *} p<, 001$ 
Tableau 3. Présentation des indices pour juger la qualité des modèles et test d'invariance $(\mathrm{N}=675)$

\begin{tabular}{cccccccc}
\hline \multicolumn{2}{c}{ Qualité du modèle initial } & \multicolumn{4}{c}{$\begin{array}{c}\text { Qualité du modèle variant } \\
\text { (en sous-groupes) }\end{array}$} & $\begin{array}{c}\text { Modèle } \\
\text { contraint }\end{array}$ & $\begin{array}{c}\text { Test } \\
\text { d'invariance }\end{array}$ \\
\hline $\begin{array}{c}\chi^{2} \\
\text { (ddl 37) }\end{array}$ & $\begin{array}{c}\text { RMSEA } \\
\text { I.C. } 90 \%\end{array}$ & CFI & $\begin{array}{c}\chi^{2} \\
\text { (ddl 74) }\end{array}$ & $\begin{array}{c}\text { RMSEA } \\
\text { I.C. } 90 \%\end{array}$ & CFI & $\begin{array}{c}\chi^{2} \\
\text { (ddl 92) }\end{array}$ & $\begin{array}{c}\text { Diff. } \chi^{2} \\
\text { (ddl 18) }\end{array}$ \\
\hline $\begin{array}{c}44,813 \\
p=0,17\end{array}$ & $\begin{array}{c}0,018 \\
0,000 \text { à } \\
0,034\end{array}$ & 0,995 & $\begin{array}{c}89,016 \\
p=0,113\end{array}$ & $\begin{array}{c}0,025 \\
0,000 \text { à } \\
0,041\end{array}$ & 0,991 & $\begin{array}{c}131,176 \\
p=0,005\end{array}$ & $\begin{array}{c}42,160 \\
p=0,001\end{array}$ \\
& & & & & \\
\hline
\end{tabular}

Tableau 4. Liens indirects spécifiques et total ainsi que le pourcentage de variance expliquée pour chaque variable médiatrice et prédite

\begin{tabular}{lrcc}
\hline Variables médiatrices & & $\mathbf{R}^{2}$ & $\begin{array}{c}\text { Liens indirects } \\
\text { spécifiques }\end{array}$ \\
\hline Chaleur parentale T2 & $\begin{array}{r}\text { Filles } \\
\text { Garçons }\end{array}$ & $\begin{array}{c}37,7 \% \\
25,4 \%\end{array}$ & $0,040^{*}$ \\
Coercition parentale T2 & Filles & $51,4 \%$ & $-0,005$ \\
& Garçons & $42,1 \%$ & 0,006 \\
Insertion sociale T2 & Filles & $20,3 \%$ & 0,015 \\
& Garçons & $17,7 \%$ & $0,033^{*}$ \\
Rejet par les pairs T2 & Filles & $26,0 \%$ & 0,010 \\
& Garçons & $14,1 \%$ & $-0,003$ \\
Difficultés scolaires T2a & Filles & $47,5 \%$ & 0,012 \\
& Garçons & $42,7 \%$ & 0,011 \\
\hline Variable prédite & & $\mathbf{R}^{2}$ & Liens indirect \\
& total \\
\hline Problèmes dépressifs T3 & Filles & $33,3 \%$ & 0,062 \\
& Garçons & $30,8 \%$ & 0,051 \\
\hline
\end{tabular}

Les valeurs de $p$ ont été calculées à partir des coefficients non standardisés.

a Il s'agit d'une échelle inversée : plus le score est faible plus les difficultés scolaires sont importantes.

${ }^{*} p<, 05 ;{ }^{* *} p<, 01 ;{ }^{* * *} p<, 001$ 


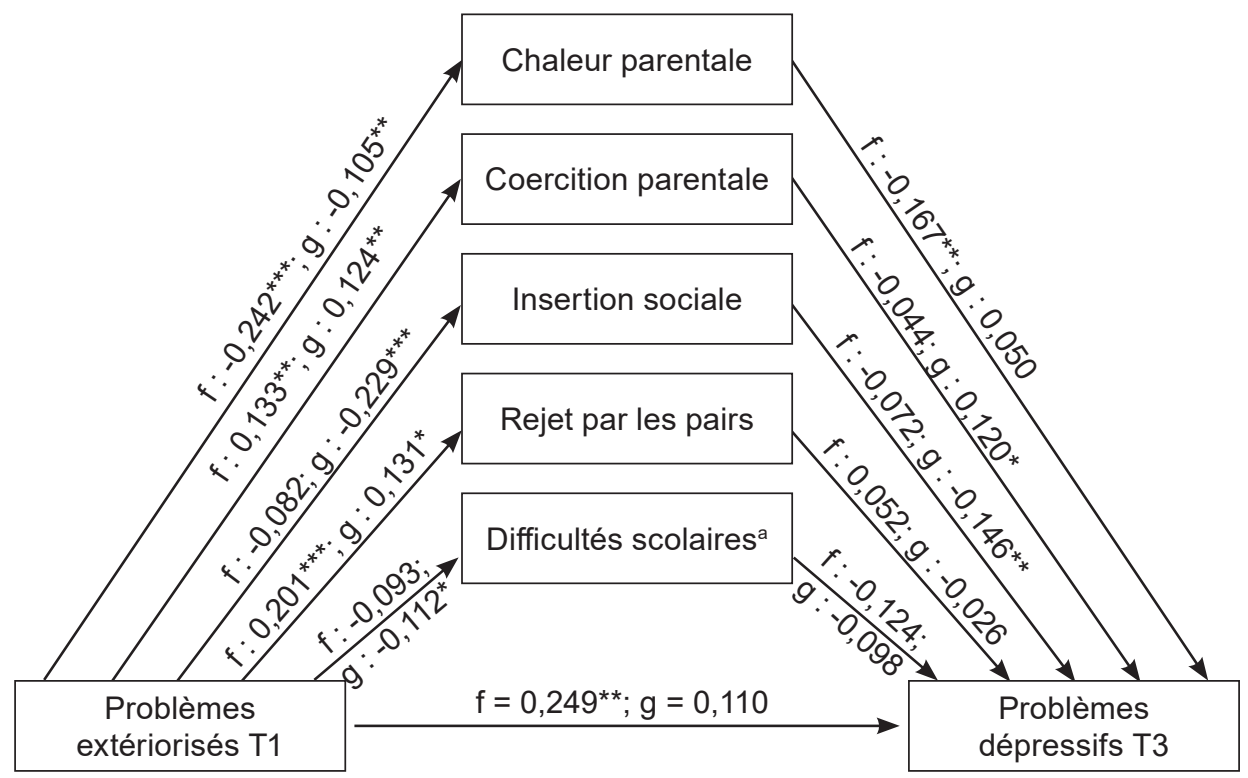

\section{Figure 2. Illustration du modèle incluant les liens directs pour les cinq médiateurs et variant selon le sexe}

Les valeurs de $p$ ont été calculées à partir des coefficients non standardisés. $f$ : valeurs des bêta standardisés pour les filles; $\mathrm{g}$ : valeurs des bêta standardisés pour les garçons. a II s'agit d'une échelle inversée : plus le score est faible plus les difficultés scolaires sont importantes.

${ }^{*} p<, 05 ;{ }^{* *} p<, 01 ;{ }^{* * *} p<, 001$

Ainsi, on remarque que chez les filles, l'effet de médiation total se traduit surtout par la qualité des interactions chaleureuses du parent auprès de son enfant. Plus particulièrement, un parent qui manque de chaleur dans son attitude auprès de son enfant mettrait les filles qui présentent des problèmes de comportement plus à risque de développer des problèmes dépressifs à l'adolescence. Pour les autres indicateurs étudiés, dont les interactions parent-enfant coercitives, la qualité de l'insertion sociale de l'enfant, le rejet par les pairs et la présence de difficultés scolaires, ils n'influencent pas de manière importante le lien entre les problèmes de comportement extériorisés et le développement de problèmes dépressifs à l'adolescence chez les filles. Comme le lien entre les problèmes extériorisés et les problèmes dépressifs chez les filles ne semble que partiellement expliqué par l'ensemble des médiateurs potentiels inclus dans le modèle total, il pourrait être intéressant de tester l'effet médiateur de d'autres indicateurs. Par exemple, selon le modèle de Patterson et Capaldi (1990), la présence de problèmes extériorisés serait associée à une plus faible estime de soi qui serait un stade antérieur au développement de la dépression. On peut donc penser que cette composante contribuerait davantage à expliquer le lien entre les problèmes de comportement à l'enfance et la dépression à l'adolescence (Patterson et Capaldi, 1990; McCann et Sato, 2000). 
L'effet total de médiation se traduit différemment chez les garçons. En effet, c'est la qualité de l'insertion sociale qui s'avère être un médiateur particulièrement déterminant du développement de problèmes dépressifs chez les garçons présentant des problèmes de comportement extériorisés. II semble que plus les garçons ayant des problèmes de comportement sont inscrits à des activités parascolaires, sont intégrés à une équipe sportive ou plus ils fréquentent plusieurs amis, moins ils sont susceptibles de développer des problèmes dépressifs à l'adolescence. On pourrait soulever l'hypothèse que la qualité de l'insertion sociale agirait comme un facteur de protection au développement de problèmes dépressifs chez les garçons, ce qui pourrait être éventuellement testé dans une future étude.

Quant à la variable du rejet par les pairs, contre toutes attentes, les résultats de la présente étude montrent que, ni chez les garçons ni chez les filles, le rejet par les pairs ne s'avère médiateur de la relation entre les problèmes de comportement extériorisés à l'enfance et les problèmes dépressifs au début de l'adolescence, du moins, en tenant compte des autres médiateurs testés dans le modèle. Pourtant, selon le modèle de Patterson et Capaldi (1990), et la prise en compte des résultats de l'étude d'Evelien et al. (2011), on se serait attendu à un effet médiateur de cet indicateur du rejet social. Des différences méthodologiques avec l'étude d'Evelien et al. (2011) peuvent possiblement expliquer ces résultats divergents. En plus de ne pas avoir testé un modèle multi médiateurs, l'étude d'Evelien et al. (2011) a été réalisé auprès d'un échantillon tiré de la population générale d'enfants beaucoup plus jeunes où la sévérité des problèmes de comportement et des problèmes dépressifs peut être à ce point faible que l'on peut questionner la validité interne de l'étude. En outre, dans l'étude d'Evelien et al. (2011), la dimension du rejet par les pairs a été évaluée directement auprès des pairs (nomination par les pairs), tandis que dans la présente étude, le rejet par les pairs a été évalué selon la perception de l'enseignant. II est possible que les pairs soient de meilleurs informateurs que les enseignants sur cette dimension. Enfin, l'influence du rejet par les pairs pour expliquer le lien entre la sévérité des problèmes extériorisés à l'enfance et le développement de problèmes dépressifs à l'adolescence mérite d'être étudiée à nouveau.

Un résultat tout autant surprenant est obtenu concernant l'absence d'un effet médiateur des difficultés scolaires. Dans le modèle de Patterson et Capaldi (1990), les difficultés scolaires sont présentées comme un médiateur important de la relation entre les problèmes de comportement et le développement de problèmes dépressifs. De plus, les études recensées sur la question de l'association entre les difficultés scolaires et les problèmes extériorisés et dépressifs laissaient aussi croire que la présence de difficultés scolaires joue un rôle important dans le développement de la dépression chez les enfants qui présentent des problèmes de comportement (Chen et Simons-Morton, 2009; Ezpeleta et al., 2005). La façon d'opérationnaliser les difficultés scolaires dans la présente étude est différente de ce qui a été fait dans les autres études. II s'agit ici d'une mesure de performance scolaire où plus le score est élevé plus l'enfant performe et plus le score est faible plus l'enfant présente des difficultés. Peut-être qu'on aurait obtenu des résultats différents en utilisant la présence ou l'absence d'échec ou de redoublement comme indicateur de difficultés. 
Quant à l'effet de la présence d'interactions parent-enfant coercitives, malgré les liens directs significatifs observés entre la sévérité des problèmes de comportement, la coercition parentale et les problèmes dépressifs, le lien de médiation n'est pas significatif ni chez les garçons ni chez les filles. Des analyses préliminaires, réalisées auprès de l'ensemble des participants avec comme seul indicateur la coercition parentale, montraient plutôt que la coercition était un médiateur significatif du lien entre les problèmes extériorisés et les problèmes dépressifs. On pourrait ainsi comprendre que la coercition parentale est un indicateur moins important une fois les autres médiateurs du modèle pris en considération.

Tel que mentionné précédemment, les résultats obtenus suggèrent d'adopter une vision plus large du modèle en portant aussi attention à la qualité des interactions parent-enfant ainsi qu'à la qualité de l'insertion sociale de l'enfant. Le rejet social ne devrait pas se limiter à l'acception ou non par les pairs. La qualité de l'implication affective du parent est inévitablement affectée par la présence des difficultés comportementales chez l'enfant (Lahey et al., 1999) et la réponse du parent aux problèmes de comportement de l'enfant vient jouer un rôle important dans le développement de la dépression (Zahn-Waxler et al., 2008). Cela montre l'importance de considérer la sphère familiale dans la mise en place de moyens d'intervention auprès des enfants présentant des problèmes de comportement afin de prévenir le développement de problèmes dépressifs.

Sur le plan des retombées pratiques, l'étude met en lumière le rôle important des relations parent-enfant dans les mécanismes menant aux problèmes dépressifs, chez les enfants présentant des problèmes de comportement extériorisé. L'intervention auprès de ces enfants devrait donc inclure un volet d'intervention auprès des parents afin de les aider à développer une relation plus chaleureuse et moins coercitive avec leur enfant, possiblement par l'emploi de pratiques éducatives positives (voir Boxer et Frick, 2008 pour des exemples de programmes). En plus d'être considérée comme une composante cruciale des pratiques probantes auprès des enfants présentant des problèmes de comportement (Boxer et Frick, 2008), l'amélioration de la relation parent-enfant pourrait prévenir des problèmes dépressifs ultérieurs, et ce, particulièrement chez les filles. En outre, souvent d'orientation cognitive comportementale, les pratiques efficaces dans le domaine des problèmes de comportement et des problèmes dépressifs prévoient des cibles communes: l'identification et la modification des pensées automatiques et des croyances à la base du maintien des problèmes (par ex. : hostilité attribuée aux autres ou dévalorisation), l'amélioration des habiletés sociales et de résolution de problèmes à mettre en pratique avec les parents et avec les pairs (Boxer et Frick, 2008; Curry et Becker, 2008). L'acquisition d'habiletés sociales semble déterminante chez les garçons, car ce faisant, on améliore leur chance de s'intégrer socialement et les risques de problèmes dépressifs ultérieurs peuvent ainsi être réduits. Travailler sur les cibles déjà connues pour réduire les problèmes de comportement tout en adaptant les contenus selon le sexe notamment en ce qui concerne l'amélioration de la relation parent-enfant et l'insertion sociale de l'enfant pourrait contribuer à prévenir la dépression à l'adolescence. 
Au-delà de son apport, la présente étude comporte certaines limites méthodologiques qui peuvent nuancer l'interprétation des résultats. D'abord, concernant l'évaluation des problèmes de comportement et des problèmes dépressifs chez les participants, bien que la présente étude ait l'avantage d'adopter une approche multi répondants (parent, enseignant, jeune), il aurait été intéressant d'avoir un tiers expert pour porter un jugement clinique global sur la sévérité des problèmes de comportement, mais aussi des problèmes dépressifs. La faisabilité de cette modalité d'évaluation étant particulièrement coûteuse, la combinaison des scores obtenus auprès de différents répondants peut être une alternative acceptable, d'autant plus que l'instrument de mesure utilisé est reconnu pour ses qualités psychométriques (Achenbach et Rescorla, 2001).

De plus, les études montrent que l'âge d'apparition de la dépression se situe généralement à l'adolescence (Ingoldsby et al., 2006). Pour la présente étude, les problèmes dépressifs furent mesurés au T3 et donc chez des enfants âgés de 10 à 13 ans se situant, pour la plupart, au tout début de l'adolescence. On peut se demander si les différences individuelles ou reliées au sexe sur le plan du développement (apparition de la puberté par exemple) ne viennent pas moduler les informations recueillies et qu'une mesure de dépression prise plus tard à l'adolescence ne donnerait pas des résultats différents. On peut aussi penser que le rejet des pairs pourrait avoir une importance plus grande chez les adolescents et les adolescentes plus âgés et que l'on pourrait observer une moins grande influence du milieu familial.

Enfin, il serait intéressant de regarder si la nature des problèmes de comportement présentés influence différemment le développement de la dépression. Une étude réalisée par Burke et al. (2010) permet de constater que ce serait particulièrement la manifestation colérique et irritable des problèmes de comportement qui prédiraient la dépression quatre ans plus tard. L'étude de Rowe, Maughan et Eley (2006) amène aussi des pistes de réflexion à ce sujet en identifiant que parmi différents types de comportements antisociaux (comportements d'opposition, agression physique, comportements délinquants), ce serait les comportements d'opposition, plus que les autres, qui auraient un lien direct sur la présence de symptômes dépressifs. Ces résultats portent à croire qu'une évaluation distincte du trouble de l'opposition et du trouble des conduites dans la mesure des problèmes de comportement pourrait donner des informations supplémentaires dans la prédiction de la dépression chez des enfants présentant des problèmes de comportement. 


\section{Références}

Achenbach, T. M. et Edelbrock, C. S. (1983). Manual for the Child Behavior Checklist and Revised Child Behavior Profile. Burlington, Vermont: University of Vermont, Department of Psychiatry.

Achenbach, T.M. et Rescorla, L.A. (2001). Manual for the ASEBA SchoolAge Forms and Profiles. Burlington, Vermont: University of Vermont, Research Centre for Children, Youth, and Families.

American Psychiatric Association (2013). Diagnostic and Statistical Manual of Mental Disorders, Fifth Edition. Arlington, VA, American Psychiatric Association, 2013.

Arcelus, J. et Vostanis, P. (2005). Psychiatric comorbidity in children and adolescents. Current Opinion In Psychiatry, 18(4), 429-434.

Beck, A. T., Sterr, R. A. et Brown, G. K. (1996). Manual for the Beck Depression Inventory-II. San Antonio, TX: Psychological Corporation.

Beekhoven, S. et Dekkers, H. (2005). Early school leaving in the lower vocational track: Triangulation of qualitative and quantitative data. Adolescence, 40(157), 197-213.

Bollen, K.A. (1989). Structural equations with latent variables. New York, NY: Wiley.

Bradshaw, C.P., Schaeffer, C.M., Petras, H. et lalongo, N. (2010). Predicting negative life outcomes from early aggressivedisruptive behavior trajectories: Gender differences in maladaptation across like domains. Journal of Youth and Adolescence, 39(8), 953-966.

Burke, J. D., Waldman, I. et Lahey, B. B. (2010). Predictive validity of childhood oppositional defiant disorder and conduct disorder: Implications for the DSM-V. Journal Of Abnormal Psychology, 119(4), 739-751.

Burt, K. B. et Roisman, G. I. (2010). Competence and psychopathology: Cascade effects in the NICHD study of early child care an youth development.
Development and Psychopathology, 22, 557-567.

Chen, R. et Simons-Morton, B. (2009). Concurrent changes in conduct problems and depressive symptoms in early adolescents: A developmental person-centered approach. Development And Psychopathology, 21(1), 285-307.

Cook, E., Pflieger, J.C., Connell, A.M. et Connell, C.M. (2015). Do specific transitional patterns of antisocial behavior during adolescence increase risk for problems in young adulthood? Journal of Abnormal Child Psychology, 43(1), 95-106.

Costello, E.J., He, J.-P., Sampson, A.A., Kessler, R.C. et Merikangas, K.R. (2014). Services for adolescents with psychiatric disorders: 12-month data from the National Comorbidity SurveyAdolescent. Psychiatric Services, 65(3), 359-366.

Coutinho, M.J., Oswald, D.P., Best, A.M. et Forness, S.R. (2002). Gender and sociodemographic factors and the disproportionate identification of culturally and linguistically diverse students with emotional disturbance. Behavioral Disorders, 27, 109-125.

Déry, M., Toupin, J., Pauzé, R. et Verlaan, P. (2004). Frequency of mental health disorders in a sample of elementary school students receiving special educational services for behavioural difficulties. Canadian Journal of Psychiatry, 49, 769-775.

Déry, M., Toupin, J., Pauzé, R. et Verlaan, P. (2005). Les caractéristiques d'élèves en difficultés de comportement placés en classe spéciale ou intégrés dans la classe ordinaire. Revue canadienne de l'éducation, 28, 1-23.

Dishion, T. J. et Kavanagh, K. (2003). Intervening in adolescent problem behavior: A family-centered approach. New York, NY: Guilford Press. 
DuPaul, G. J., Rapport, M. D. et Perriello, L. M. (1991). Teacher ratings of academic skills: The development of the Academic Performance Rating Scale. School Psychology Review, 20(2), 284-300.

Evelien, E. C., van Lier, P. C., Stegge, H., Terwogt, M. et Koot, H. M. (2011). The development of conduct problems and depressive symptoms in early elementary school children: The role of peer rejection. Journal Of Clinical Child And Adolescent Psychology, 40(2), 245-253.

Ezpeleta, L., Granero, R. et Doménech, J. M. (2005). Differential contextual factors of comorbid conduct and depressive disorders in Spanish children. European Child and Adolescent Psychiatry, 14(5), 282-291.

Fanti, K. A. et Henrich, C. C. (2010). Trajectories of pure and co-occurring internalizing and externalizing problems from age 2 to age 12: Findings from the National Institute of Child Health and Human Development Study of Early Child Care. Developmental Psychology, 46(5), 1159-1175.

Hu, L. T. et Bentler, P. M. (1999). Cutoff criteria for fit indexes in covariance structure analysis: Conventional criteria versus new alternatives. Structural Equation Modeling: A Multidisciplinary Journal, 6(1), 1-55.

Ingoldsby, E. M., Kohl, G. O., McMahon, R. J. et Lengua, L. (2006). Conduct Problems, Depressive Symptomatology and Their Co-Occurring Presentation in Childhood as Predictors of Adjustment in Early Adolescence. Journal Of Abnormal Child Psychology: An Official Publication Of The International Society For Research In Child And Adolescent Psychopathology, 34(5), 603-621.

Kazdin, A.E. (2005). Parent management training: Treatment for oppositional, aggressive, and antisocial behaviour in children and adolescents. Oxford, NY: Oxford University Press.

Keenan, K. et Hipwell, A. E. (2005). Preadolescent clues to understanding depression in girls. Clinical Child And
Family Psychology Review, 8(2), 89105.

Kessler, R. C., Avenevoli, S. et Merikangas, K. R. (2001). Mood disorders in children and adolescents: An epidemiologic perspective. Biological Psychiatry, 49, 1002-1014.

Khaleque, A. et Rohner, R.-P. (2001). Perceived parental acceptancerejection and psychological adjustment: A meta-analysis of cross-cultural and intracultural studies. Journal of Marriage and Family, 64(1), 54-64.

King, S., Waschbusch, D. A., Frankland, B. W., Andrade, B. F., Thurston, C. M., McNutt, L. et Terrio, B. (2005). Taxonomic Examination of ADHD and Conduct Problem Comorbidity in Elementary School Children Using Cluster Analyses. Journal Of Psychopathology And Behavioral Assessment, 27(2), 77-88.

Lahey, B., Waldman, I. et McBurnett, K. (1999). The development of antisocial behavior: An integrative causal model. Journal Of Child Psychology And Psychiatry [serial online]. July 1999;40(5).

Lahey, B. B. et Waldman, I. D. (2003). A developmental propensity model of the origins of conduct problems during childhood and adolescence. Dans B. B. Lahey, T. E. Moffitt, A. Caspi (dir). Causes of conduct disorder and juvenile delinquency (pp. 76-117). New York, NY US: Guilford Press.

Lane, K.L. (2003). Identifying young students at risk for antisocial behavior: The utility of "teachers as tests". Behavior Disorders, 28, 360-369.

Moffitt, T. E. (1993). Adolescence-limited and life-course-persistent antisocial behavior: A developmental taxonomy. Psychological Review, 100(4), 674-701.

Moffit, T. E. (2006). Life-course persistent versus adolescence-limited antisocial behaviour. Dans Cicchetti, D. et Cohen, D.J. (dir.). Developmental psychopathology, vol3: Risk, disorder, and adaptation (2 ${ }^{\text {nd }}$ ed). (p.570-598). Hoboken: NJ. John Wiley and Sons Inc. 
Muthén, L. K. et Muthén, B. O. (1998-2014). Mplus User's Guide. Los Angeles, CA: Muthén and Muthén.

Patterson, G. R. (1986). Performance models for antisocial boys. American Psychologist, 41(4), 432-444.

Patterson, G. R. et Capaldi, D. M. (1990). A mediational model for boys' depressed mood. Dans J. E. Rolf, A. S. Masten, D. Cicchetti, K. H. Nuechterlein, S. Weintraub (dir), Risk and protective factors in the development of psychopathology (pp. 141-163). New York, NY US: Cambridge University Press.

Reinecke, M. A. (1995). Comorbidity of conduct disorder and depression among adolescents: Implications for assessment and treatment. Cognitive And Behavioral Practice, 2(2), 299-326.

Rockhill, C. M., Stoep, A., McCauley, E. et Katon, W. J. (2009). Social competence and social support as mediators between comorbid depressive and conduct problems and functional outcomes in middle school. Journal of Adolescence, 32(3), 535-553.

Rohner, R. P. (2005). Parental acceptancerejection/control questionnaire (PARQ/ control): Test manual. Dans RP Rohner et A. Khaleque (dir.), Handbook for the study of parental acceptance and rejection (pp. 137-186). Storrs, CT: Rohner research publications.

Roskam, I., Kinoo, P. et Nassogne, M. C. (2007). L'enfant avec troubles extemalisés du comportement: approche épigénétique et développementale. Neuropsychiatrie de l'enfance et de l'adolescence, 55(4), 204-213.

Rowe. R., Costello, E.J., Angold, A., Copeland, W.E. et Maughan, B. (2010). Developmental Pathways in oppositionnal defiant disorder and conduct disorder. Journal of Abnormal Psychology, 119(4), 726-738.
Stringaris, A., Lewis, G. et Maughan, B. (2014). Developmental pathways from childhood conduct problems to early adult depression: findings from the ALSPAC cohort. The British Journal of Psychiatry, 205(1), 17-23.

Valla, J., Bergeron, L., Breton, J., Gaudet, N., Berthiaume, C., St-Georges, M. et al. (1997). Enquête québécoise sur la santé mentale des jeunes de 6 à 14 ans: 1992-volume 1: Méthodologie. Hôpital Rivière-des-Prairies et Santé Québec en collaboration avec le Ministère de la Santé et des Services Sociaux. Montréal.

van Lier, P. A. C., Vitaro, F., Barker, E. D., Bendgen, M., Tremblay, R. E. et Boivin, M. (2012). Peer victimization, poor academic achievement, and the link between childhood externalizing and internalizing problems. Child Development, 83(5), 1775-1788.

Waschbusch, D. A. (2002). A meta-analytic examination of comorbid hyperactiveimpulsive-attention problems and conduct problems. Psychological Bulletin, 128(1), 118-150.

Wolff, J. C. et Ollendick, T. (2006). The comorbidity of conduct problems and depression in childhood and adolescence. Clinical Child And Family Psychology Review, 9(3-4), 201-220.

Zahn-Waxler, C., Shirtcliff, E. A. et Marceau, K. (2008). Disorders of childhood and adolescence: Gender and psychopathology. Annual Review of Clinical Psychology, 42, 75-303. 